\title{
Multi-Detector STEM-EDS Mapping of Ion-Irradiated Nanostructured Ferritic Alloys
}

\author{
Chad M. Parish, ${ }^{1}$ Ryan M. White, ${ }^{2}$ James M. LeBeau, ${ }^{2}$ Yanwen Zhang, ${ }^{1}$ and Michael K. Miller ${ }^{1}$ \\ 1. Materials Science and Technology Division, Oak Ridge National Laboratory, Oak Ridge, TN, USA \\ 2. Materials Science and Engineering Department, North Carolina State University, Raleigh, NC, USA
}

Materials used in advanced fission or fusion reactors must be able to withstand radiation damage doses of several hundred displacements per atom (dpa) under high temperature and elevated creep stresses, in corrosive environments. Because actual reactors can only apply a few dpa/year of neutron irradiation to specimen capsules, heavy ion irradiation at dose rates of hundreds of dpa/day are useful for rapid screening of candidate alloys. Heavy ions produce a damaged region of a few thousand nanometers from the surface of a test specimen and this dose profile results in large damage gradients in the material volume. To characterize the damage regions, an FEI Titan ChemiSTEM scanning transmission electron microscope with an aberration-corrected probe and large-area, four-sensor X-ray detector, was used to

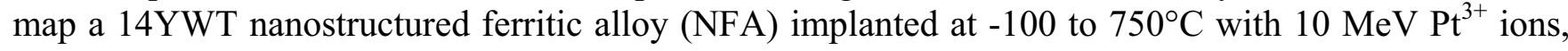
with a peak damage depth of $\sim 1000 \mathrm{~nm}$ and a damage tail extending to $\sim 1500 \mathrm{~nm}$. By rapidly mapping $>500 \times 2000 \mathrm{~nm}$ regions, the high brightness probe and high-efficiency detector of the ChemiSTEM permitted the NFA response over the full damage range (i.e., zero to peak to zero dpa) to be recorded.

FIB-prepared surface cross-sections prepared from the as-fabricated and irradiated NFAs were mapped using the following parameters: $6-7 \mathrm{nA}, \approx 0.5 \mathrm{~nm}, 200 \mathrm{keV}$ corrected probe and $252 \times 1000$ pix, $528 \times 2078 \mathrm{~nm}$ spectrum images acquired over $3600 \mathrm{sec}$. This $\approx 2 \mu \mathrm{m}$ deep map captures the entire radiation response of the NFA, extending from the surface to the maximum dpa (at $\sim 1000 \mathrm{~nm}$ ) and back to zero dpa (at a depth of $\sim 1500 \mathrm{~nm}$ ) [1]. For the highest fluence specimen $\left(4 \times 10^{16} \mathrm{Pt} / \mathrm{cm}^{2}\right)$, the peak dpa is $\sim 320$ and the SRIM-estimated peak Pt is $\sim 1$ at. \%. Maps for the as-fabricated NFA, irradiated $4 \times 10^{16} \mathrm{Pt} / \mathrm{cm}^{2}$ at $-100^{\circ} \mathrm{C}$, and $4 \times 10^{16} \mathrm{Pt} / \mathrm{cm}^{2}$ at $750^{\circ} \mathrm{C}$, are shown in Fig. 1. The as-fabricated NFA shows $\mathrm{Cr}$ and $\mathrm{W}$ segregation to the grain boundaries (GB), Ti-rich $\sim 50 \mathrm{~nm}$ precipitates [2] dispersed in the matrix, and Ti-Y-enriched particles at the GBs. In the $-100^{\circ} \mathrm{C}$ specimen, the 1 at. $\%$ peak Pt implantation profile is clear, and matches the SRIM prediction. Above $30 \mathrm{dpa}$, all GB segregation and Ti-Y GB precipitates are homogenized, and the larger Ti-rich particles are blurred by ballistic mixing into the matrix [1]. The $750^{\circ} \mathrm{C}$ specimen (close to proposed fusion reactor service conditions) retains $\mathrm{Cr}$ and $\mathrm{W}$ segregation and indicates growth of the Ti-Y-O particles at the GBs. This behavior points to a selfhealing process active at $750^{\circ} \mathrm{C}$ but not at $-100^{\circ} \mathrm{C}$, indicating $14 \mathrm{YWT}$ is promising for in-reactor service.

Combining a high brightness aberration-corrected probe with high-efficiency X-ray detectors allow the entire response of heavy-ion-irradiation specimens to be studied in a single map, which was a previously impossible experiment.

[1] C. M. Parish et al., J. Nucl. Mater., V418 (2011) 106.

[2] M. K. Miller et al., Mater. Sci. Tech., V27 (2011) 729.

[3] Research supported by the U.S. Department of Energy (DOE), Office of Basic Energy Sciences (BES), Materials Sciences and Engineering Division, and through a user project supported by ORNL's Shared Research Equipment (ShaRE) User Program, which is also sponsored by DOE-BES. Ion irradiations performed using the Environmental Molecular Sciences Laboratory (EMSL), a national scientific user facility, which is sponsored at Pacific Northwest National Laboratory by the Office of 
Biological and Environmental Research, US DOE. Titan ChemiSTEM usage supported by NCSU Analytical Instrumentation Facility.

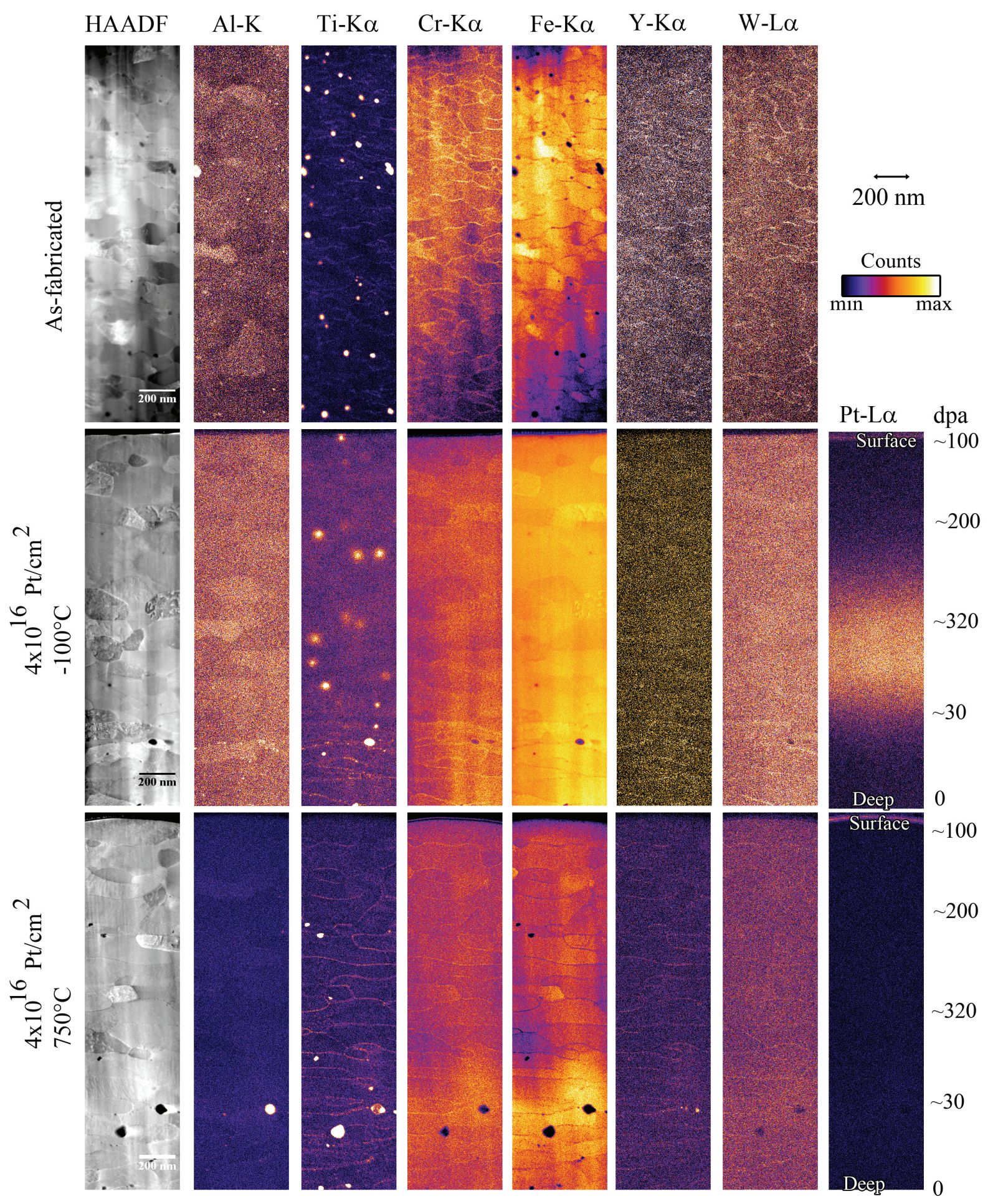

Figure 1. As-fabricated, high-fluence Pt ion irradiated at $-100^{\circ} \mathrm{C}$ and $750^{\circ} \mathrm{C}$ maps of $14 \mathrm{YWT}$ NFA. 